\title{
A DEEPLY ECLIPSING DETACHED DOUBLE HELIUM WHITE DWARF BINARY
}

\author{
S. G. Parsons ${ }^{1}$, T. R. Marsh ${ }^{1}$, B. T. Gänsicke ${ }^{1}$, A. J. DraKe ${ }^{2}$, and D. Koester ${ }^{3}$ \\ ${ }^{1}$ Department of Physics, University of Warwick, Coventry, CV4 7AL, UK \\ ${ }^{2}$ California Institute of Technology, 1200 E. California Blvd, CA 91225, USA \\ ${ }^{3}$ Institut für Theoretische Physik und Astrophysik, Universität Kiel, Germany \\ Received 2011 April 15; accepted 2011 May 17; published 2011 June 17
}

\begin{abstract}
Using Liverpool Telescope+RISE photometry we identify the $2.78 \mathrm{hr}$ period binary star CSS 41177 as a detached eclipsing double white dwarf binary with a $21,100 \mathrm{~K}$ primary star and a $10,500 \mathrm{~K}$ secondary star. This makes CSS 41177 only the second known eclipsing double white dwarf binary after NLTT 11748. The 2 minute long primary eclipse is $40 \%$ deep and the secondary eclipse $10 \%$ deep. From Gemini+GMOS spectroscopy, we measure the radial velocities of both components of the binary from the $\mathrm{H} \alpha$ absorption line cores. These measurements, combined with the light curve information, yield white dwarf masses of $M_{1}=0.283 \pm 0.064 M_{\odot}$ and $M_{2}=0.274 \pm 0.034 M_{\odot}$, making them both helium core white dwarfs. As an eclipsing, double-lined spectroscopic binary, CSS 41177 is ideally suited to measuring precise, model-independent masses and radii. The two white dwarfs will merge in roughly $1.1 \mathrm{Gyr}$ to form a single sdB star.
\end{abstract}

Key words: binaries: eclipsing - stars: individual (CSS 41177) - white dwarfs

\section{INTRODUCTION}

There are of order 100-300 million close pairs of white dwarfs in the Galaxy. They are thought to be the dominant source of background gravitational waves detectable by LISA (Hils et al. 1990). The loss of orbital angular momentum via gravitational radiation in these systems will eventually bring the two white dwarfs into contact with one another. Those that achieve this within a Hubble time are possible progenitors of AM CVn binaries, R CrB stars, and Type Ia supernovae (Iben \& Tutukov 1984). The coalescence of two low-mass, helium core white dwarfs is also a possible formation channel for the creation of single sdB stars (Webbink 1984); indeed it may be one of the more important sdB formation channels (Han et al. 2003). There are now 43 detached double white dwarf binaries known (Kilic et al. 2011; Marsh 2011, and references therein) but few of these yield the precise parameters needed to determine their eventual fate.

The subject of this Letter, CSS 41177 (SDSS J100559.10+ 224932.2), was listed as a white dwarf plus main-sequence binary by Drake et al. (2010). They used a marginal 2MASS detection to infer the presence of a late-type companion though they note that a small faint object could also produce the observed transit signal. We observed CSS 41177 as part of a monitoring campaign for period variations in white dwarf plus main-sequence binaries. With a better sampled eclipse and detection of the secondary eclipse, we realized that it was in fact a detached eclipsing double white dwarf binary, making it only the second known after NLTT 11748 (Steinfadt et al. 2010), but in this case the secondary star contributes $22 \%$ of the overall flux (compared to $3 \%$ in NLTT 11748). Follow-up spectroscopy revealed that CSS 41177 was also a double-lined spectroscopic binary.

Here we present Liverpool Telescope (LT)+RISE photometry and Gemini+GMOS ${ }^{4}$ spectroscopy of CSS 41177, and use these observations, and mass-radius relations, to determine the system

\footnotetext{
4 Gemini Multi-Object Spectrograph.
}

parameters. We also discuss the evolution and eventual fate of the binary.

\section{OBSERVATIONS AND THEIR REDUCTION}

\subsection{LT+RISE Photometry}

Three primary eclipses and two secondary eclipses of CSS 41177 were observed using the LT and RISE (Steele et al. 2004) between 2011 February and April. RISE is a high-speed frame transfer CCD camera with a single wideband $V+R$ filter (Steele et al. 2008). All observations were made in $2 \times 2$ binning mode, giving a scale of 1.1 arcsec pixel ${ }^{-1}$, and with exposure times of $12-13 \mathrm{~s}$.

The raw data are automatically run through a pipeline that debiases, removes a scaled dark frame, and flat-fields the data. The source flux was determined with aperture photometry using a variable aperture, whereby the radius of the aperture is scaled according to the FWHM, using the ULTRACAM pipeline (Dhillon et al. 2007). Variations in observing conditions were accounted for by determining the flux relative to the nearby star G 54-11 ( $V=14.6)$.

\subsection{Gemini+GMOS Spectroscopy}

CSS 41177 was observed using GMOS on the $8 \mathrm{~m}$ Gemini Observatory North telescope (Hook et al. 2004) on 2011 March 15 . We used the R831 grating with a $0.5 \operatorname{arcsec}$ slit and $4 \times 2$ binning (spatial by spectral) to give a resolution of $R \sim 4400$ centered at $\mathrm{H} \alpha$. We used an exposure time of 10 minutes, recording 17 spectra covering an entire orbit.

The average bias and flat-field correction were carried out using the FIGARO package from STARLINK and nightly bias and tungsten frames. We optimally extracted each spectrum (Marsh 1989) and used a CuAr arc lamp to establish an accurate wavelength scale. The arc spectrum was fitted with a fifth-order polynomial to 15 lines which gave an rms of 0.05 pixel. The individual spectra were normalized to the continuum level using a polynomial fit to the continuum regions and the spectra were placed onto a heliocentric wavelength scale. 

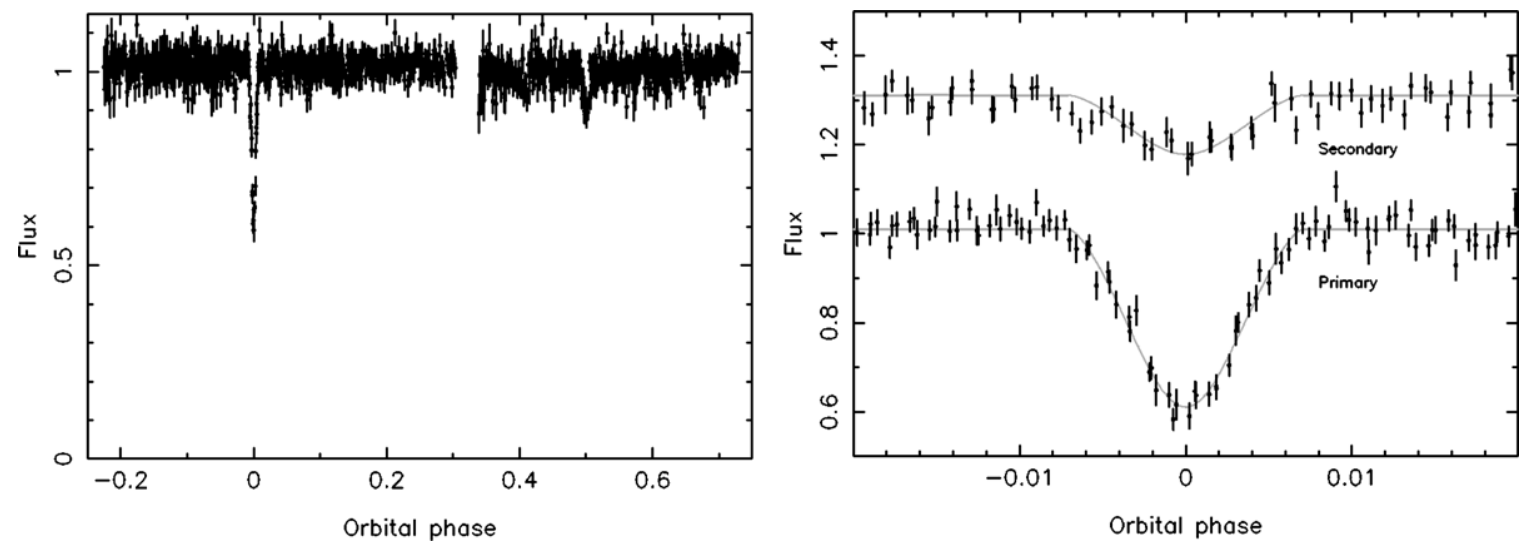

Figure 1. Left: LT+RISE photometry of CSS 41177 folded on its $2.78 \mathrm{hr}$ orbital period and binned by a factor of two. Right: the unbinned primary and secondary eclipse data. The secondary eclipse is shifted back by half a cycle and vertically by 0.3 .

Table 1

Mid-eclipse Times

\begin{tabular}{lcc}
\hline \hline $\begin{array}{l}\text { Cycle } \\
\text { Number }\end{array}$ & $\begin{array}{l}\text { Eclipse Time } \\
\text { MJD(BTDB) }\end{array}$ & $\begin{array}{r}\text { Uncertainty } \\
\text { MJD(BTDB) }\end{array}$ \\
\hline-171 & 55599.087788 & 0.000013 \\
0 & 55618.926463 & 0.000016 \\
302 & 55653.963116 & 0.000020 \\
\hline
\end{tabular}

\section{RESULTS}

\subsection{Light Curve Model Fitting}

The left-hand panel of Figure 1 shows the LT+RISE photometry of CSS 41177 folded on its $2.78 \mathrm{hr}$ orbital period and binned by a factor of two. Since both eclipses are detected, the light curve information alone constrains the system inclination and the radii scaled by the binary separation.

To measure the system parameters we fitted the light curve using a code written to produce models for the general case of binaries containing a white dwarf (see Copperwheat et al. 2010 for details). The program subdivides each star into small elements with a geometry fixed by its radius as measured along the direction of centers toward the other star. From an initial set of parameters defined by the user, the code produces model light curves which are initially fitted to the RISE data using Markov chain Monte Carlo (MCMC) minimization to produce a set of covariances. We then ran another MCMC minimization using these covariances to define the parameter jumps. To produce the final parameters and their errors, we followed the procedures described in Collier Cameron et al. (2007). The parameters needed to define the model were: the mass ratio, $q=M_{2} / M_{1}$, the inclination, $i$, the radii scaled by the binary separation, $R_{1} / a$ and $R_{2} / a$, the surface brightness ratio, $S_{1} / S_{2}$, quadratic limbdarkening coefficients for both the white dwarfs, the time of mid eclipse, $T_{0}$, and the period, $P$.

We corrected all the times to Barycentric Dynamical Time (TDB). We initially fitted each light curve individually in order to measure mid-eclipse times for the three primary eclipses recorded. The second eclipse recorded was set to cycle number zero. Our measured eclipse times are shown in Table 1. The zero point of the ephemeris and the period were allowed to vary in our final fit. We kept the mass ratio fixed as 1.0 (the light curves of this well-detached system are independent of the mass ratio). We determined the quadratic limb-darkening coefficients from a pair of white dwarf atmosphere models with temperatures of $21,100 \mathrm{~K}$ and $10,500 \mathrm{~K}$ and $\log g=7.3$ (see Section 3.2) folded through the RISE filter profile (Gänsicke et al. 1995). We determined coefficients of $a=0.105$ and $b=0.228$ for the primary and $a=0.176$ and $b=0.288$ for the secondary for $I(\mu) / I(1)=1-a(1-\mu)-b(1-\mu)^{2}$, where $\mu$ is the cosine of the angle between the line of sight and the surface normal; we kept these values fixed. The inclination, scaled radii, and surface brightness ratio were allowed to vary.

The right-hand panel of Figure 1 shows the best fit to the RISE photometry around the eclipses. The two eclipses constrain the system inclination to $89^{\circ} .2 \pm 0.3$. The constraints on the two scaled radii are shown in the left-hand panel of Figure 3. We also update the ephemeris to

$$
\operatorname{MJD}(\mathrm{BTDB})=55618.926447(9)+0.11601549(5) E,
$$

which is consistent with the ephemeris of Drake et al. (2010).

\subsection{Temperatures}

We used data from the Sloan Digital Sky Survey (SDSS) to determine the temperatures of the two white dwarfs in CSS 41177. Initially, we fitted its SDSS spectrum as a single white dwarf using the models of Koester et al. (2005), which results in a $T_{\text {eff, } 1}=21,535 \pm 214 \mathrm{~K}$ and $\log g=7.36 \pm 0.44$. To determine the temperature of both stars better, we fitted the SDSS ugriz magnitudes with a composite of two DA white dwarf models, with the additional constraint of the surface brightness ratio $S_{1} / S_{2}=2.90 \pm 0.23$ in the LT/ RISE band, as measured from the light curve. This fit results in $T_{\text {eff, } 1}=21,100 \pm 800 \mathrm{~K}$ and $T_{\text {eff, } 2}=10,500 \pm 500 \mathrm{~K}$, where the quoted errors are estimated, as they correlate with the uncertainties in the surface gravities. The best-fit composite model reproduces well the observed Galaxy Evolution Explorer $(G A L E X)$ ultraviolet fluxes.

\subsection{Radial Velocities}

The left-hand panel of Figure 2 shows the trailed spectrum of CSS 41177 around the $\mathrm{H} \alpha$ line. The non-LTE absorption core from the primary (hotter) star is clearly visible. There is also a weaker absorption component moving in anti-phase with the absorption from the primary star originating from the secondary (cooler) star. No other features are visible in the GMOS spectra.

Initially we fitted just the primary star's absorption. For each spectrum, we fitted the line using a combination of a straight line plus a Gaussian. However, the radial velocity 

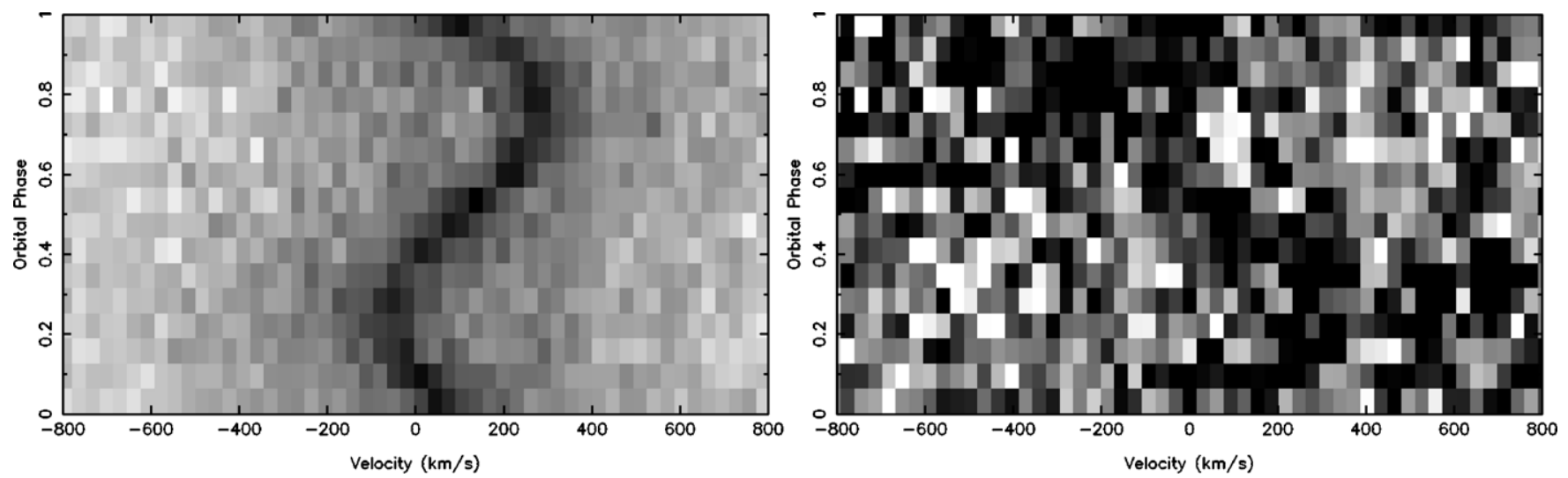

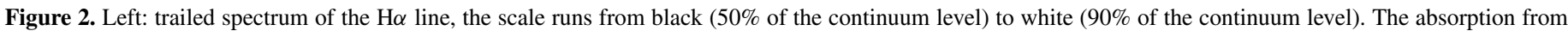

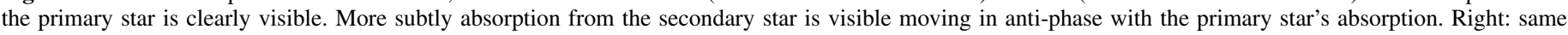

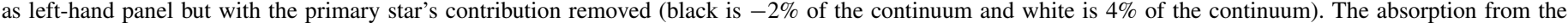
secondary star is now visible.
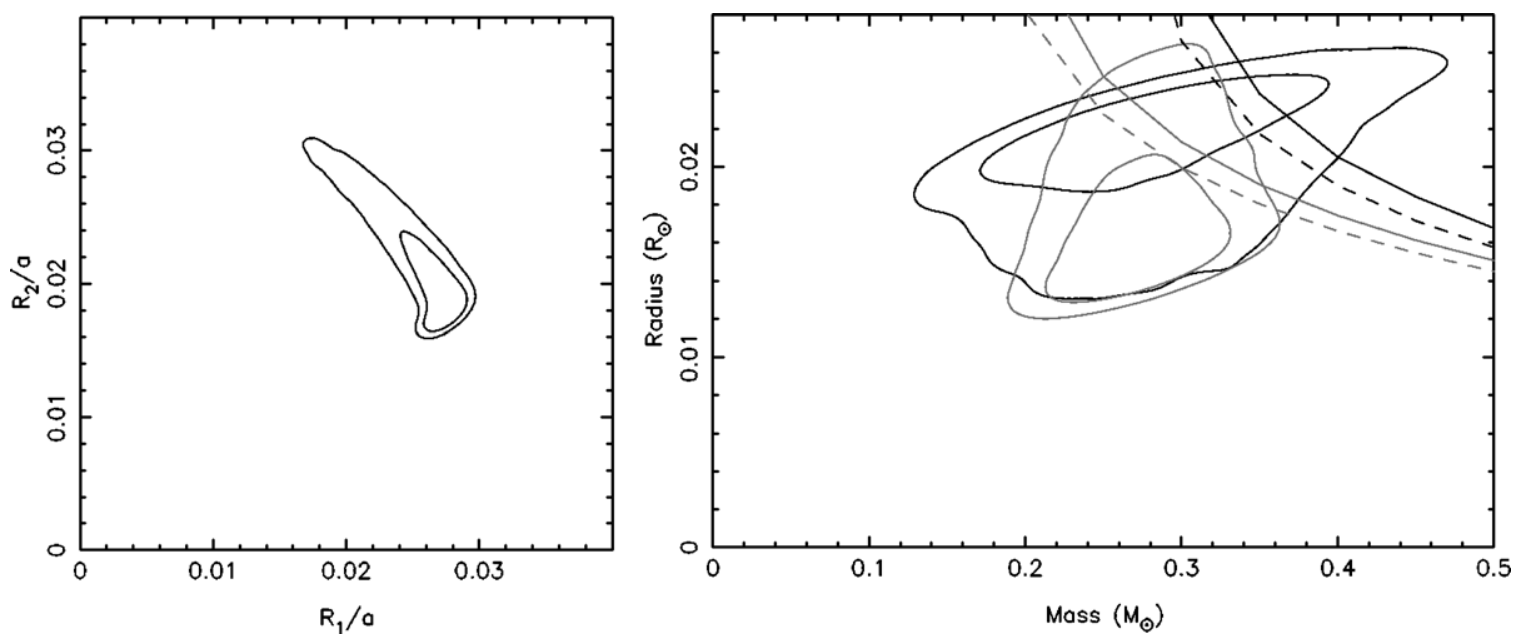

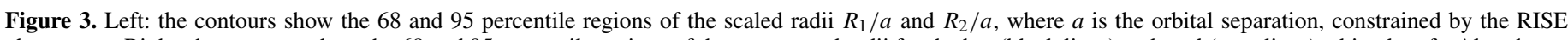

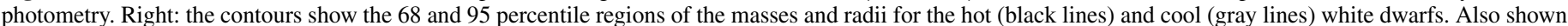

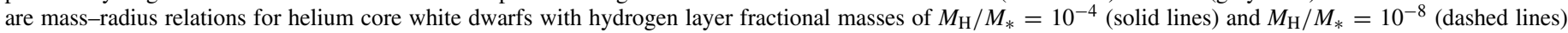

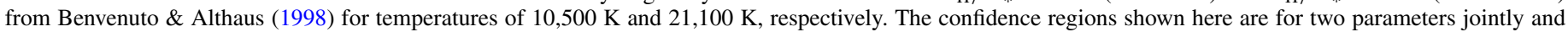
should not be directly compared to the uncertainties in Table 2, which are more closely related to single parameter confidence intervals.

measured does not represent the true radial velocity of the primary star as the absorption from the secondary star causes a slight underestimation. Nevertheless, we used these velocities to correct out the motion of the primary star. We then averaged the shifted spectra to obtain the rest-frame spectrum of the primary star which we then subtracted from all the observed spectra. The result is shown in the right-hand panel of Figure 2, clearly showing the absorption from the secondary star.

We fitted the absorption from the secondary star in the same way as the primary star. This too underestimated the true velocity since the primary star's contribution had not been completely removed. However, both fits provide us with an initial starting point for $K_{1}$ and $K_{2}$, the radial velocity amplitudes of both stars. In order to measure accurate radial velocities, both absorption components need to be fitted simultaneously. We fit both lines together by simultaneously fitting all of the spectra. We used a combination of a straight line and Gaussians for each spectrum (including a broad Gaussian component to account for the wings of the primary star's absorption) and allowed the position of the Gaussians to change velocity according to

$$
V=\gamma+K \sin (2 \pi \phi)
$$

for each star, where $\gamma$ is the offset in the line from its rest wavelength and $\phi$ is the orbital phase of the spectrum. Due to the faintness of the secondary star's absorption we keep the offset $\gamma_{2}=\gamma_{1}$ to reduce the number of degrees of freedom. All other parameters were initially set to those measured from fitting the lines individually. We fitted the data using Levenberg-Marquardt minimization (Press et al. 1986). The resultant fit had a reduced $\chi^{2}$ of 1.02 . We find $K_{1}=$ $177 \pm 3 \mathrm{~km} \mathrm{~s}^{-1}, K_{2}=181 \pm 20 \mathrm{~km} \mathrm{~s}^{-1}$, and $\gamma=109 \pm 2 \mathrm{~km} \mathrm{~s}^{-1}$. These values imply that CSS 41177 is an equal mass binary (or very close to, given the uncertainty on $K_{2}$ ).

\subsection{System Parameters}

The RISE photometry constrains $i, R_{1} / a$, and $R_{2} / a . K_{1}$ and $K_{2}$ are constrained from the spectroscopy, therefore we can determine the masses and radii of both white dwarfs using Kepler's third law,

$$
\frac{G\left(M_{1}+M_{2}\right)}{a^{3}}=\frac{4 \pi^{2}}{P^{2}},
$$


Table 2

Stellar and Binary Parameters for CSS 41177

\begin{tabular}{lccc}
\hline \hline Parameter & Value & Parameter & Value \\
\hline R.A. & $10: 05: 59.11$ & $K_{1}\left(\mathrm{~km} \mathrm{~s}^{-1}\right)$ & $177 \pm 3$ \\
Decl. & $+22: 49: 32.3$ & $K_{2}\left(\mathrm{~km} \mathrm{~s}^{-1}\right)$ & $181 \pm 20$ \\
GALEX FUV & $16.72 \pm 0.04$ & $T_{\text {eff, } 1}(\mathrm{~K})$ & $21100 \pm 800$ \\
GALEX NUV & $16.98 \pm 0.03$ & $T_{\text {eff }, 2}(\mathrm{~K})$ & $10500 \pm 500$ \\
$u$ & $17.317 \pm 0.010$ & $M_{1}\left(M_{\odot}\right)$ & $0.283 \pm 0.064$ \\
$g$ & $17.266 \pm 0.005$ & $M_{2}\left(M_{\odot}\right)$ & $0.274 \pm 0.034$ \\
$r$ & $17.612 \pm 0.006$ & $R_{1}\left(R_{\odot}\right)$ & $0.0210 \pm 0.0026$ \\
$i$ & $17.899 \pm 0.008$ & $R_{2}\left(R_{\odot}\right)$ & $0.0174 \pm 0.0031$ \\
$z$ & $18.151 \pm 0.024$ & $a\left(R_{\odot}\right)$ & $0.821 \pm 0.048$ \\
$d$ (pc) & $350 \pm 13$ & $i(\mathrm{deg})$ & $89.2 \pm 0.3$ \\
$P_{\text {orb }}$ (days) & $0.1160156(1)$ & & \\
\hline
\end{tabular}

where $P$ is the orbital period, given the orbital separation from

$$
\frac{2 \pi}{P} a \sin i=K_{1}+K_{2} \text {. }
$$

We determined the masses and radii for each model produced in the MCMC analysis of the light curve using the measured radial velocities and Kepler's laws. The right-hand panel of Figure 3 shows the regions of allowed masses and radii for both white dwarfs based only upon the photometric and spectroscopic constraints. Also shown are models of helium core white dwarfs with hydrogen layer fractional masses of $M_{\mathrm{H}} / M_{*}=10^{-4}$ (solid lines) and $M_{\mathrm{H}} / M_{*}=10^{-8}$ (dashed lines) from Benvenuto \& Althaus (1998) for temperatures of 10,500 K (gray) and $21,100 \mathrm{~K}$ (black) which, given the large uncertainties in our measurements, are consistent with the measured masses and radii. The final system parameters are listed in Table 2.

\section{CONCLUSIONS}

We have discovered that CSS 41177 is a detached, eclipsing, double white dwarf binary containing two low-mass helium core white dwarfs with masses of $M_{1}=0.283 \pm 0.064 M_{\odot}$ and $M_{2}=0.274 \pm 0.034 M_{\odot}$ and radii of $R_{1}=0.0210 \pm 0.0026 R_{\odot}$ and $R_{2}=0.0174 \pm 0.0031 R_{\odot}$. The hotter white dwarf has a temperature of $21,100 \pm 800 \mathrm{~K}$ and the cooler white dwarf has a temperature of $10,500 \pm 500 \mathrm{~K}$ placing it on the red edge of the instability strip.

CSS 41177 is ideally set up to measure precise modelindependent masses and radii for both white dwarfs and thus can be used to test white dwarf mass-radius relations. To date only one white dwarf has had its mass and radius independently measured to high precision (NN Ser; Parsons et al. 2010), therefore CSS 41177 offers two additional white dwarfs which can test mass-radius relations at the lower mass range.

Since the white dwarfs in CSS 41177 have roughly equal masses they will eventually undergo a merger resulting in the formation of a single $\sim 0.6 M_{\odot}$ sdB star (Han et al. 2003). This will occur in roughly $1.1 \mathrm{Gyr}$ due to the loss of orbital angular momentum via gravitational radiation. The regular eclipse times from CSS 41177 should make it an excellent timing standard for optical astronomy, provided there are no unseen companions.

Balmer/Lyman lines in the models were calculated with the modified Stark broadening profiles of Tremblay \& Bergeron (2009), kindly made available by the authors. T.R.M and B.T.G acknowledge support from the Science and Technology Facilities Council (STFC) grant number ST/F002599/1. The Liverpool Telescope is operated on the island of La Palma by Liverpool John Moores University in the Spanish Observatorio del Roque de los Muchachos of the Instituto de Astrofisica de Canarias with financial support from the UK Science and Technology Facilities Council. This work is based on observations obtained at the Gemini Observatory under program ID GN-2011A-DD-1, which is operated by the Association of Universities for Research in Astronomy, Inc., under a cooperative agreement with the NSF on behalf of the Gemini partnership: the National Science Foundation (United States), the Science and Technology Facilities Council (United Kingdom), the National Research Council (Canada), CONICYT (Chile), the Australian Research Council (Australia), Ministério da Ciência e Tecnologia (Brazil), and Ministerio de Ciencia, Tecnología e Innovación Productiva (Argentina).

Facilities: Liverpool:2m, Gemini:Gillett

\section{REFERENCES}

Benvenuto, O. G., \& Althaus, L. G. 1998, MNRAS, 293, 177

Collier Cameron, A., et al. 2007, MNRAS, 380, 1230

Copperwheat, C. M., Marsh, T. R., Dhillon, V. S., Littlefair, S. P., Hickman, R., Gänsicke, B. T., \& Southworth, J. 2010, MNRAS, 402, 1824

Dhillon, V. S., et al. 2007, MNRAS, 378, 825

Drake, A. J., et al. 2010, arXiv:1009.3048

Gänsicke, B. T., Beuermann, K., \& de Martino, D. 1995, A\&A, 303, 127

Han, Z., Podsiadlowski, P., Maxted, P. F. L., \& Marsh, T. R. 2003, MNRAS, 341, 669

Hils, D., Bender, P. L., \& Webbink, R. F. 1990, ApJ, 360, 75

Hook, I. M., Jørgensen, I., Allington-Smith, J. R., Davies, R. L., Metcalfe, N., Murowinski, R. G., \& Crampton, D. 2004, PASP, 116, 425

Iben, I., Jr., \& Tutukov, A. V. 1984, ApJS, 54, 335

Kilic, M., Brown, W. R., Allende Prieto, C., Agüeros, M. A., Heinke, C., \& Kenyon, S. J. 2011, ApJ, 727, 3

Koester, D., Rollenhagen, K., Napiwotzki, R., Voss, B., Christlieb, N., Homeier, D., \& Reimers, D. 2005, A\&A, 432, 1025

Marsh, T. R. 1989, PASP, 101, 1032

Marsh, T. R. 2011, Class. Quantum Grav., 28, 094019

Parsons, S. G., Marsh, T. R., Copperwheat, C. M., Dhillon, V. S., Littlefair, S. P., Gänsicke, B. T., \& Hickman, R. 2010, MNRAS, 402, 2591

Press, W. H., Flannery, B. P., \& Teukolsky, S. A. 1986, Numerical Recipes. The Art of Scientific Computing (Cambridge: Cambridge Univ. Press)

Steele, I. A., Bates, S. D., Gibson, N., Keenan, F., Meaburn, J., Mottram, C. J., Pollacco, D., \& Todd, I. 2008, Proc. SPIE, 7014, 70146J

Steele, I. A., et al. 2004, Proc. SPIE, 5489, 679

Steinfadt, J. D. R., Kaplan, D. L., Shporer, A., Bildsten, L., \& Howell, S. B. 2010, ApJ, 716, L146

Tremblay, P.-E., \& Bergeron, P. 2009, ApJ, 696, 1755

Webbink, R. F. 1984, ApJ, 277, 355 